CHAPITRE 3

\title{
Obésité et diabète de type 2
}

JEAN-PAUL RIOU 



\section{Résumé}

La recherche dans le domaine de l'obésité et du diabète de type 2 a été marquée, durant les quinze dernières années, par quatre événements majeurs :

1. La reconnaissance d'une "explosion » de la fréquence de l'obésité, maladie de l'adaptation aux changements de l'environnement hygiénodiététique (diminution de l'activité physique; augmentation de la densité alimentaire, diminution du coût des calories). Cette épidémie touche notamment les milieux les plus défavorisés et les enfants. Son apparition, associée au vieillissement de la population, est à l'origine du nombre croissant de patients atteints de diabète de type 2: 180 millions en 2005 de par le monde, 330 millions prévus en $2025 \ldots$

2. La reconnaissance des fonctions endocrines du tissu adipeux. Celui-ci $\mathrm{n}^{\prime}$ apparaît plus comme un simple réservoir énergétique indispensable en cas de famine mais comme un tissu complexe, sécrétant de vraies hormones (leptine, résistine, adiponectine...) et aussi des protéines proinflammatoires (interleukine 6, tumor necrosis factor alpha...). Ces dernières sont probablement impliquées dans l'apparition du diabète de type 2 mais aussi dans les complications associées à l'obésité : hypertension artérielle, infarctus du myocarde, cancer de l'utérus et de la prostate.

3. Le contrôle neuro-hormonal de la prise alimentaire et ou de la dépense énergétique a révélé toute sa complexité. II met en cause des signaux périphériques endocriniens (insuline, leptine, hormones thyrö̈diennes, cortisol), métaboliques (glucose, acides gras libres) et digestifs (cholecystokinine, ghréline...). Ces signaux sont intégrés au niveau hypothalamique par un réseau complexe de neurones sécrétant peptides orexigènes ou anorexigènes (PYY, MSH, CRF, CART...). Tous ces peptides sont maintenant parfaitement caractérisés. Leurs fonctions ont été précisées grâce à de nombreux modèles de souris transgéniques. Des obésités " génétiques 》 chez l'homme ont été caractérisées notamment en France.

4. Le substratum génétique du diabète de type 2 (90\% de concordance chez les jumeaux homozygotes) est en passe d'être compris. Les diabètes de type MODY (autosomique dominant) sont maintenant définis et phénotypés. Le mécanisme du diabète de type 2 avec obésité androïde, caractérisé par une insulinorésistance majeure et un déficit de l'insulinosécrétion probablement précoce, reste encore mystérieux mais les progrès de la génétique et de l'épigénétique devraient rapidement déboucher sur une compréhension des mécanismes de cette pathologie.

La France, à travers I'Inserm, l'Inra, le CNRS, l'Agence française de sécurité sanitaire des aliments et produits de santé, l'ANR et les ministères de la Santé et 
de la Recherche, a su mettre en place, à la fin des années 1980, une vraie politique associant financement de la recherche, mesures de santé publique (programme national nutrition santé) et plus récemment soutien des réseaux diabète et obésité par la Sécurité sociale.

Ces engagements, au service de l'excellence de la recherche, ont permis à plusieurs équipes (Paris, Lyon, Toulouse, Lille...) d'être des leaders mondiaux dans leurs domaines.

Il est proposé que cette politique soit renforcé notamment en :

- créant de petites équipes de pointe en liaison forte avec l'industrie pharmaceutique;

- lançant un grand projet de prévention primaire de l'obésité andrö̈de chez l'enfant et/ou dans les milieux défavorisés;

- favorisant des regroupements humains et technologiques sur les thèmes : nutrition, cerveau et imagerie métabolique;

- toutes ces mesures seront sans effet si les organismes publics impliqués n'arrivent pas à simplifier l'organigramme du financement de la recherche et le travail administratif du chercheur.

\section{Introduction}

Le diabète touche environ 180 millions de personnes de par le monde et la majorité d'entre elles présente un diabète de type 2 . Ce nombre déjà considérable devrait augmenter à 330 millions d'ici 2025. Cette augmentation spectaculaire de la fréquence du diabète de type 2 est parallèle à l'augmentation alarmante de l'obésité qui est un facteur de risque majeur de diabète de type 2. Cette double épidémie est maintenant largement reconnue et les organisations nationales et internationales commencent à prendre en compte les aspects non seulement scientifiques mais aussi économiques et sociétaux de cette pathologie.

Les quelques éléments présentés ci-dessous n'ont pas pour objectifs de présenter une revue exhaustive des recherches en cours dans ce domaine mais plutôt de faire le point sur certains aspects scientifiques et médicaux de la recherche, notamment française qui pourraient favoriser de nouveaux éclairages et susciter, de la part des pouvoirs publics, de nouvelles approches aussi bien scientifiques que médicales, voire économiques et politiques. 


\section{1 | L'obésité}

\section{1 Épidémiologie}

L'obésité, définie dans le monde entier comme un indice de masse corporelle (poids/taille $\left(\mathrm{m}^{2}\right)$ ) supérieur ou égal à 30 , augmente depuis vingt ans dans presque tous les pays et pas exclusivement les pays riches. En France, $10 \%$ des adultes et $16 \%$ des enfants sont obèses. Cette prévalence reste inférieure à celle des États-Unis (30\%) et à celles des pays de l'ex-Europe de l'Est (20\%). Deux données épidémiologiques nouvelles doivent être soulignées :

- l'augmentation de la prévalence est surtout importante chez les enfants, conduisant à l'apparition de diabète de type 2 chez l'enfant. Il s'agit là $d^{\prime}$ 'une pathologie qui était inexistante il y a seulement vingt ans ;

- l'obésité est plus frappante dans les populations les plus défavorisées. Les données épidémiologiques françaises et nord-américaines montrent que la carte de l'obésité et du diabète de type 2 se superpose à la carte de la pauvreté. Cette relation est lourde de sens et témoigne de l'impact des facteurs éducatifs et socio-économiques au point que l'on en vient à se demander si l'obésité ne serait pas d'abord un problème économique avant de devenir un problème métabolique.

\subsection{Une maladie de l'adaptation}

La reconnaissance de l'obésité comme une maladie de l'adaptation aux changements de notre environnement (diminution de l'activité physique, augmentation de la densité alimentaire et diminution "du coût des calories ") est très récente.

En France, ce n'est qu'en 1988 que la nutrition est devenue une discipline médicale universitaire à part entière. L'immense majorité des médecins praticiens n'ont eu, actuellement, aucune formation, aucun enseignement digne de ce nom sur cette pathologie, l'obésité, qui est devenue ce jour la première cause de mortalité aux États-Unis du fait de son association avec le diabète de type 2, les pathologies cardiovasculaires et le cancer (corps de l'utérus, prostate...). Malgré ce constat sévère, cette pathologie, notamment en France, est heureusement devenue l'objet d'une attention soutenue non seulement des instituts de recherche mais aussi du ministère de la Santé et des leaders socio-économiques des industries agroalimentaires. Plusieurs éléments de nature scientifique ont contribué à cette évolution récente. 


\subsection{Le tissu adipeux (TA) : un tissu complexe aux fonctions multiples}

Jusqu'en 1994, le TA apparaît comme un " organe simple » ayant pour fonction de stocker l'énergie en situation d'abondance et de la libérer en situation de carence énergétique. Depuis cette époque, il est apparu que le tissu adipeux produit des signaux endocriniens et des adipokines, facteurs impliqués non seulement dans la régulation de la prise alimentaire mais aussi dans les pathologies associées à l'obésité : insulinorésistance, syndrome métabolique, athérosclérose.

\subsubsection{L'adipocyte : "cellule endocrinienne"}

Le rôle " endocrinien » de l'adipocyte a été révélé par la découverte de la leptine, identifié par clonage positionnel, comme le produit du gène $\mathrm{Ob}$ responsable d'une forme monogénique d'obésité chez la souris et chez l'homme. La leptine est une cytokine de 164 AA sécrétée par l'adipocyte dans la circulation sanguine qui agit à distance sur des récepteurs, notamment hypothalamiques. La leptine étant produite proportionnellement à la masse du tissu adipeux, son rôle pourrait être d'informer le cerveau de l'état des réserves, où elle exerce un rétrocontrôle négatif de la prise alimentaire et positif de la dépense énergétique. L'identification chez l'animal et chez l'homme d'obésités monogénique par mutation du gène de la leptine ou de son récepteur a définitivement donné ses nouveaux quartiers de noblesse au tissu adipeux. Ce nouveau paradigme apparaît comme l'élément déterminant de la création de plusieurs groupes de recherche française sur les liens entre obésité et génétique (Paris, Lille) et entre obésité et insulinorésistance (Lyon-Toulouse).

Depuis dix ans, les travaux sur l'adipocyte ont conduit à identifier de nombreuses autres fonctions endocriniennes du tissu adipeux. Parmi celles-ci, on peut souligner le rôle du tissu adipeux dans la transformation de la cortisone inactive en hydrocortisone active. Cette interconversion périphérique existe dans le foie mais aussi dans le tissu adipeux, notamment abdominal. Elle est assurée par la 11 -bêła-hydroxystérö̈de-déshydrogénase de type 1. Cette enzyme, et les cofacteurs qui lui sont associés, pourraient être impliqués dans la genèse du syndrome métabolique. Les souris $\mathrm{KO}$ pour ce gène sont résistantes au diabète de type 2 induit par un régime riche en graisse alors que les souris surexprimant ce gène dans le tissu adipeux abdominal présentent un « hypercorticisme abdominal » avec diabète de type 2 et insulinorésistance sévère. Ces travaux et d'autres, notamment chez l'homme, ont conduit à l'étude qualitative du rôle des différents dépôts de tissu adipeux dans le corps. Il apparaît que le tissu adipeux 
abdominal, du fait de sa situation et de ses propriétés spécifiques (lipolyse augmentée, production de cytokines, sensibilité à l'insuline) pourrait jover un rôle critique dans la genèse du diabète de type 2 associé à l'obésité.

\subsubsection{Adipokines, insulinorésistance et pathologies cardiovasculaires associées à l'obésité}

L'injection de leptine recombinante chez des souris "lipoatrophiques 》 ou chez des souris obèses déficientes en leptine, améliore la sensibilité à l'insuline indépendamment de toute variation du poids ou de la prise alimentaire. Cela est en contradiction avec le fait que les patients obèses qui ont une leptinémie augmentée sont insulinorésistants. Il est probable que l'effet insulinosensibilisateur de la leptine est atteint pour des taux d'hormonémie minimale.

L'adiponectine, découverte en 1995, est une protéine de 247 acides aminés sécrétés spécifiquement par l'adipocyte mature. Il s'agit du transcrit le plus abondant de l'adipocyte. Elle circule sous des formes moléculaires variées, à des concentrations importantes : 5 à $20 \mathrm{mg} / \mathrm{l}$. À l'inverse de la plupart des adipokines, l'expression et les concentrations plasmatiques d'adiponectine sont diminuées dans la situation d'insulinorésistance. Chez les souris, dans les modèles $d^{\prime}$ insulinorésistance et/ou de diabète de type 2, l'injection d'adiponectine inverse le processus d'insulinorésistance et améliore la tolérance glucidique. Cette action bénéfique s'accompagne d'une forte augmentation de l'oxydation des acides gras dans le muscle squelettique et d'une potentialisation de l'action de l'insuline sur la production hépatique de glucose. Enfin, l'adiponectine possède des propriétés anti-inflammatoires et athérogènes, au moins in vitro.

D'autres adipocyłokines ont été plus récemment encore impliquées dans la genèse de l'insulinorésistance et des complications cardiovasculaires du diabète de type 2 et de l'obésité andrö̈de. Le tissu adipeux humain produit des quantités importantes d'IL- 6 dont le rôle dans l'insulinorésistance est controversé même si sa concentration plasmatique est corrélée positivement au poids corporel et négativement à la sensibilité à l'insuline. La résistine et le TNF- $\alpha$ ont été aussi impliqués sans que leur rôle précis chez l'homme dans la pathogénie du syndrome métabolique ait été démontré.

L'ensemble de ces études a ouvert tout un champ d'investigation sur les relations entre tissu adipeux, système immunitaire et athérome. La protéine $C$ réactive dont la synthèse par le foie est contrôlée en partie par l'interleukine 6 et le TNF- $\alpha$ sécrété par le tissu adipeux, est aujourd'hui clairement reconnue comme un facteur de risque cardiovasculaire. Le type et la localisation du tissu adipeux péri-artériel (presque absent autour des artères des membres supérieurs) pourraient jouer un rôle majeur dans la localisation des phénomènes 
athérothrombotiques sur certains vaisseaux. II apparaît ainsi que le tissu adipeux joue un rôle différent en fonction de sa localisation. De plus, les études très récentes montrent que ce tissu est hétérogène. Les cellules immunocompétentes en son sein commencent à être identifiées, caractérisées et impliquées dans la régulation du métabolisme et des fonctions du tissu adipeux.

L'émergence de ces nouveaux concepts a conduit à réévaluer la notion de " cross-talk » interorganes. Il apparaît ce jour clairement que le métabolisme du tissu adipeux retentit sur le métabolisme musculaire indépendamment de la sécrétion d'insuline et de l'intensité de la lipolyse. L'activation du facteur de transcription PPAR $\gamma$ exprimé quasiment uniquement dans le tissu adipeux, $\mathrm{s}^{\prime}$ accompagne d'une augmentation de la sensibilité à l'insuline du muscle. Le message en retour du muscle vers le tissu adipeux a été aussi clairement démontré sur des modèles de souris $\mathrm{KO}$ tissu spécifique du récepteur à l'insuline.

\subsection{Relations entre obésité abdominale, pathologies cardiovasculaires et diabète de type 2}

Elles ont été parfaitement identifiées au niveau clinique à la fin des années 1940 par l'équipe du professeur J. Vague, précurseur en France des travaux cliniques et scientifiques sur l'obésité. Néanmoins, il a fallu attendre les années 1980 et l'apparition du concept d'insulinorésistance pour que ce syndrome pathologique soit reconnu. On considère classiquement que l'obésité gynoïde, surcharge pondérale de la partie inférieure du corps, est essentiellement source de complications mécaniques : syndrome d'apnée du sommeil, hypertension artérielle pulmonaire avec insuffisance respiratoire, gonarthrose et lombalgies, thromboses veineuses profondes, complications obstétricales... À l'inverse, l'obésité andrö̈de, surcharge pondérale prédominante sur la partie supérieure du corps, notamment l'abdomen, est associée à des complications métaboliques endocriniennes et cardiovasculaires: diabète de type 2, dyslipidémie, hypertension artérielle, insuffisance coronaire, accidents vasculaires cérébraux, infertilité, altération de l'hémostasie... Cette nosologie, particulièrement pertinente en pratique médicale quotidienne pour dépister les sujets à risque de diabète et d'HTA par exemple, a été remplacée progressivement par le terme "syndrome métabolique ", syndrome regroupant au moins trois critères des complications des obésités androïdes. En fait, plusieurs définitions du syndrome métabolique ont vu le jour sous la pression plus ou moins consciente des épidémiologistes et de l'industrie pharmaceutique. On estime que 10 à $15 \%$ de la population adulte française pourrait rentrer dans l'une ou l'autre des définitions du syndrome métabolique. On voit mal, dans ces conditions, l'industrie pharmaceutique baisser les bras dans la perspective d'un tel marché préventif. On voit aussi assez mal les pouvoirs publics s'engager dans le financement 
de thérapeutiques pour une telle population alors que les mesures hygiénodiététiques ont fait la preuve de leur efficacité non seulement dans le traitement du diabète de type 2 associé à l'obésité androïde mais aussi dans la prévention de l'évolution de l'obésité andrö̈de vers le diabète de type 2. Des conflits scientifiques d'envergure ont probablement assez souvent servi d'alibi à des problématiques économiques ayant une toute autre dimension. Ces conflits ont conduit très récemment les autorités diabétologiques nord-américaines et européennes à prendre une position diamétralement opposée en remettant en cause l'intérêt scientifique et clinique du concept de syndrome métabolique. À notre avis, une telle évolution est regrettable pour des raisons médicales et scientifiques. Au niveau médical, le concept d'obésité andröde (et non pas de syndrome métabolique) permet de définir, par la simple mesure du tour de taille, une entité clinique susceptible de guider utilement les choix du médecin dans la définition d'une stratégie thérapeutique. Au niveau scientifique, la caractéristique commune à tous les éléments de l'obésité androïde et/ou du syndrome métabolique est d'être associée à une insulinorésistance. Plusieurs groupes associant chercheurs et médecins (Paris, Nice, Lyon. ..) d'envergure internationale ont contribué à déchiffrer les mécanismes moléculaires de cette résistance à l'action de l'insuline tant au niveau du muscle qu'au niveau du tissu adipeux. Des modifications profondes de l'état de phosphorylation des protéines impliquées dans la transmission du message insulinique ont été démontrées tant chez l'animal que chez l'homme. Ces altérations biochimiques persistent sur des modèles de cellules musculaires humaines cultivées in vitro. La question non résolue à ce jour est de savoir si l'insulinorésistance présente chez l'obèse, et notamment chez l'obèse andrö̈le, est adaptative ou constitutionnelle. Les études chez les apparentés non diabétiques de parents présentant un diabète de type 2 suggèrent que l'insulinorésistance précède durant de longues années l'apparition du diabète suggérant que l'insulinorésistance pourrait être primitive (constitutionnelle?). Cette hypothèse mériterait $d^{\prime}$ être confirmée. Il est aussi possible, mais ce n'est pas le paradigme ambiant, que l'insulinorésistance soit, dès le début, une réponse de l'organisme pour protéger les tissus d'un stockage sans fin d'un excès de calories glucidiques et/ou lipidiques. En faveur d'une telle hypothèse, il faut insister sur l'existence d'une hypersensibilité à l'insuline dans la phase précoce de l'obésité chez l'enfant. L'insulinorésistance, dans cette hypothèse, ne deviendrait pathogène que parce qu'elle redirigerait les substrats énergétiques vers le foie et les cellules endothéliales des vaisseaux, favorisant alors la stéatose hépatique et l'athérosclérose.

\subsection{Rôle du cerveau dans la régulation de l'appétit et de la dépense énergétique}

Le cerveau joue un rôle clé dans la régulation de l'appétit et de la prise alimentaire. Si les cliniciens ont, depuis longtemps, analysé cette évidence 
(anorexie mentale, boulimie, obésité secondaire aux effets des neuroleptiques, développement $d^{\prime}$ 'antagonistes des récepteurs aux cannabinoïdes pour contrôler le poids corporel), ce n'est que dans les dix dernières années que le rôle central de l'hypothalamus a été, au moins en partie, décrypté. l'hypothalamus reçoit des signaux de la périphérie de nature hormonale et métabolique. Les influx transmis par le nerf vague envoient les informations viscérales telles que la distension gastrique, la mobilité intestinale. Les signaux hormonaux et métaboliques sont multiples (leptine, insuline, cortisol, ghréline et cholécystokinine, glucose, acides gras). La clarification des rôles respectifs de chacun des noyaux de l'hypothalamus reste mal élucidée, notamment du fait de la redondance des différents systèmes orexigènes et anorexigènes. Néanmoins, le décryptage de la signalisation leptinique a rapidement progressé du fait de travaux essentiellement nord-américains. La leptine, en se liant à son récepteur, inhibe l'activité des neurones synthétisant deux peptides orexigéniques (neuropeptide $Y$ et agouti related peptide) et active les neurones synthétisant deux peptides anorexigéniques (alpha-melanocyte stimulating hormone : $\alpha M S H$ et cocaine amphetamine related transcript : CART). L'augmentation de la production locale de $\alpha \mathrm{MSH}$ stimule le récepteur MC4R qui déclenche un signal d'arrêt de la prise alimentaire tant chez l'homme que chez le rat ou la souris. Les mutations des gènes codant la leptine, le récepteur de la leptine, la propopiomélanocortine (précurseur de l'aMSH), le MC4R, conduisent à une obésité monogénique chez l'animal et chez l'homme. La fréquence significative de cette pathologie dans l'origine de l'obésité morbide a été clairement établie par plusieurs équipes, françaises notamment.

Les signaux hormonaux périphériques sont nombreux. La cholécystokinine diminue la prise alimentaire et agit de façon synergique avec la leptine. La ghréline, sécrétée au niveau de l'estomac et du cerveau, stimule la prise alimentaire et sa sécrétion est inhibée à la suite d'un repas. L'insuline contrôle, au niveau central, le poids corporel comme le montre l'obésité (modérée) des souris KO pour le récepteur à l'insuline dans le cerveau. Néanmoins, l'hypoglycémie résultant d'un excès d'insuline (diabète type de 1 sous insuline, insulinome pancréatique) entraîne une faim incoercible et un gain de poids.

Récemment, on a montré que l'augmentation de la néoglucogenèse intestinale secondaire à un régime riche en protéines générait une hyperglycémie, exclusivement portale, qui signale au cerveau via le nerf vague de réduire la prise alimentaire. Ces découvertes sur l'interaction intestin-cerveau dans le contrôle de la prise alimentaire renforcent l'hypothèse d'un rôle crucial de la glycémie portale comme élément central des mécanismes de l'intégration métabolique au niveau cérébral de l'état nutritionnel. 


\section{6 Évaluation des politiques scientifiques, de santé publique et des industries agroalimentaires face à l'épidémie d'obésité}

Si les chercheurs se sont résolument engagés dans de nouvelles approches d'étude sur l'obésité depuis une quinzaine d'années, l'environnement politicoéconomique d'organisation de la recherche et des soins a largement favorisé ce mouvement.

L'engagement du ministère de la Recherche et de l'Enseignement supérieur en faveur de la nutrition à la fin des années 1980, sous l'impulsion, notamment, d'équipes parisiennes et lyonnaises, a été un starter efficace car il s'est immédiatement inscrit dans la durée. On a pu ainsi rapidement développer l'enseignement de la nutrition et créer progressivement 4 groupements d'intérêt public : centre de recherche en nutrition humaine (Clermont-Ferrand, Lyon, Nantes, Paris). Ce mouvement, fortement soutenu par I'Inra, I'Inserm et les centres hospitalo-universitaires, a été un élément déterminant dans la création d'interfaces médicales et scientifiques entre les biochimistes, les physiologistes, les médecins, les $\mathrm{CHU}$, les vétérinaires et les industries agroalimentaires. La recherche en nutrition a aussi grandement bénéficié de la création de l'Afssaps qui a permis d'insérer les choix médicaux et scientifiques dans l'ensemble du tissu socio-économique national et européen. Plus récemment, l'engagement résolu des acteurs de la recherche et des structures de soins sur l'obésité a débouché sur de grands programmes nationaux: Programme national nutrition santé, réseaux médicaux de soins des obèses et des diabétiques, loi de Santé publique 2004 sur les boissons et aliments sucrés, mobilisation des acteurs des caisses Assurances maladie, programme spécifique de l'Agence nationale de la recherche, création du Département d'Alimentation humaine à l'Inra prenant en compte l'aliment, de sa fabrication à son utilisation chez l'homme, et intégrant les aspects sociétaux et économiques des choix alimentaires des Français.

L'ensemble de ces actions a permis de structurer quelques pôles forts reconnus au niveau international et contribuant, de façon majeure, aux actions visant à comprendre, soigner et prévenir l'obésité. Il est intéressant de noter que les politiques volontaristes de l'Inra d'un côté et les politiques incitatrices de l'Inserm se sont retrouvées, grâce à quelques personnalités clés dans les deux organismes, pour travailler en commun et promouvoir des recommandations conséquentes dans le domaine de la santé publique. Un tel mouvement a été très contraignant, au bon sens du terme, pour les industries agroalimentaires qui, de plus en plus, intègrent lentement, dans leur politique commerciale, les enjeux de la santé publique. 
Cette évolution doit maintenant s'étendre à une dimension européenne qu'il faut à tout prix aborder. La Politique agricole commune a été mise en place pour soutenir le revenu des agriculteurs. La santé des consommateurs n'a donc jamais fait explicitement partie des objectifs de cette politique, malgré ses effets en terme de santé. Il faut maintenant prendre conscience que les choix de la Pac pèsent lourdement (!) sur les choix alimentaires des consommateurs. Les choix actuels vont dans le sens d'une réduction du soutien octroyé aux agriculteurs par les prix. II en résulte que les prix des produits auparavant soutenus (céréales, sucre, lait, beurre et viande bovine) devraient diminuer. Au contraire, les viandes de porc, de volaille, les fruits, les légumes et les huiles végétales n'étaient pas ou peu soutenus via les prix de marché. Ils ne peuvent donc que se renchérir en valeur relative. Une telle évolution va à l'encontre des attentes santé du Programme national nutrition santé en induisant un renchérissement supplémentaire des fruits et légumes par rapport aux sucres et aux produits laitiers. Ainsi, le levier de la Pac devrait, en prenant en compte ses effets santé, amener les consommateurs à s'orienter plus sur les fruits et légumes que sur les produits laitiers et sucrés.

\section{2 | Le diabète}

\subsection{Classification, évolution de la prise en charge et de la recherche}

Le diabète est défini par la constatation le matin à jeun de deux glycémies sur sang veineux $\geqslant a ̀$ a $1,27 \mathrm{~g} / \mathrm{l}$. Cette définition, acceptée et reconnue dans le monde entier, regroupe des entités nosologiques très différentes. On distingue schématiquement parmi les 2,5 millions de diabétiques connus en France : le diabète de type $1(10 \%)$, le diabète de type $2(80$ à $85 \%)$ et le diabète que I'on appelait autrefois intermédiaire (5-10\%).

Nous ne traiterons pas ici du diabète de type 1 qui résulte de la destruction, par le système immunitaire, des cellules bêta (insulinosécrétrices) des îlots de Langerhans du pancréas. La présence d'une insulite, la détection d'autoanticorps spécifiques sur coupes de pancréas humain, la caractérisation de lymphocytes T activés contre des antigènes exprimés par la cellule bêta, font de ce diabète 1 une maladie auto-immune dont les mécanismes sont hors du propos de ce rapport centré sur les relations diabète de type 2-nutrition-obésité.

Les diabètes dits "intermédiaires 》 regroupent plusieurs entités comme les diabètes secondaires (pancréatite, traitement corticoödes...), les diabètes monogéniques (Maturity Onset Diabetes of the Youth : MODY), les diabètes mitochondriaux et les diabètes auto-immuns de l'âge adulte (LADA). 
Enfin, le diabète de type 2 survient, en général, vers $45-50$ ans chez des sujets obèses avec une prédominance notable de la surcharge graisseuse au niveau abdominal et/ou viscéral. Sa fréquence devrait augmenter de façon majeure dans les vingt prochaines années du fait du vieillissement de la population et de la persistance d'une iatrogenèse hygiénodiététique. De plus, le développement de thérapeutiques préventives dans le domaine cardiovasculaire, de thérapeutiques efficaces sur l'évolution de la néphropathie diabétique, de coordination des soins du diabète entre les médecins praticiens, les spécialistes et les $\mathrm{CHU}$ à travers, notamment, les réseaux de soins, contribuent largement à l'allongement considérable de l'espérance de vie des patients atteints de diabète de type 2. Cette évolution très perceptible actuellement, devrait conduire à une large réévaluation des stratégies thérapeutiques et de l'organisation des soins en diabétologie dans les établissements publics et privés.

Tous ces états pathologiques ont en commun d'entraîner à long terme, du fait, notamment, de l'hyperglycémie, des complications micro-angiopathiques et macro-angiopathiques graves expliquant que le diabète soit la première cause de cécité chez l'adulte avant 65 ans dans les pays développés, la première cause d'insuffisance rénale après 50 ans, une cause majeure d'amputation, $d^{\prime}$ accidents coronariens et d'accidents vasculaires cérébraux.

La prise en compte, par les pouvoirs politiques, de l'importance du diabète de type 2 comme pathologie majeure des années actuelles et à venir est, somme toute, assez récente. Il a fallu attendre 1996 pour que la Direction générale de la santé publie une directive sur l'organisation des soins des diabétiques. Cette directive est loin d'être mise en œuvre dans toutes les régions françaises. En fait, c'est le surcoût médical et social du diabète, surcoût né de la mise en œuvre de thérapeutiques efficaces, qui a contraint les pouvoirs publics à réagir.

Les organismes de recherche tant français qu'européens ou nord-américains ont été longtemps inspirés dans leur politique par une culture endocrinologique et " gènes candidat. » Cette culture a conduit à développer, peut-être à l'excès, des modèles animaux transgéniques ou non, induisant un diabète qui $a$, le plus souvent (pour le diabète de type 2), peu de points communs avec le diabète de type 2 de l'espèce humaine. Ces travaux ont néanmoins permis de beaucoup mieux cerner les mécanismes génétiques, biochimiques et cellulaires pouvant engendrer une hyperglycémie. On assiste, actuellement, faute de moyen pertinent d'études quantitatives chez l'homme, à une accumulation chez l'animal d'informations scientifiques sur les "diabétogènes 》 et à un appauvrissement des connaissances vraiment pertinentes sur les mécanismes conduisant au diabète de type 2. La physiologie du diabète de type 2 reste, à ce jour, pour le moins confuse. 


\subsection{Physiopathologie du diabète de type 2 : le paradigme}

Le diabète de type 2 est une maladie multifactorielle résultant de l'interaction de facteurs génétiques et environnementaux. Deux mécanismes sont en cause dans la genèse du diabète de type 2 chez l'homme :

- une insulinorésistance qui s'exprime au niveau du muscle et du tissu adipeux par la diminution de l'action stimulatrice de l'insuline sur le transport du glucose et au niveau du foie par une diminution de l'action inhibitrice de l'insuline sur la production hépatique de glucose;

- une diminution de l'insulinosécrétion qui devient, au fil du temps, incapable de compenser l'insulinorésistance.

Classiquement, I'insulinorésistance est première, le défaut insulinosécrétoire secondaire. Nous verrons plus loin que rien n'est moins sûr. Ce schéma explicatif, bien qu'admis globalement depuis vingt ans, n'est pas, aujourd'hui, démontré de façon formelle. Le développement considérable des modèles $\mathrm{d}^{\prime} \mathrm{in}$ sulinorésistance par $\mathrm{KO}$ de très nombreux gènes a contribué de façon majeure à notre compréhension des mécanismes de régulation de la glycémie mais $\mathrm{n}^{\prime} \mathrm{a}$, en rien, permis de confirmer cette physiopathologie du diabète de type 2 chez I'homme. Chacun des éléments de ce dogme sera discuté pas à pas sachant qu'à ce jour, aucune théorie satisfaisante ne permet d'embrasser toute la complexité des mécanismes à l'origine du diabète de type 2.

\subsection{Génétique, diabète de type 2 et environnement}

L'existence de diabète monogénique autosomique dominant est connue de longue date mais ce n'est que depuis douze ans que plusieurs équipes, notamment françaises, ont identifié les gènes en cause : glucokinase, hepatic nuclear factor $1 \alpha, 4 \alpha$, insulin promotor factor 1 , neurogenic differentiating factor 1 , peroxisome proliferator activated receptor $\gamma$ (PPAR $\gamma$ ). Comme souligné ci-dessus, ces gènes ne concernent que 2 à $4 \%$ de l'ensemble des patients présentant un diabète de type 2. Ces travaux ont donné lieu à la prolifération de différentes souris KO chez lesquelles d'innombrables gènes candidat ont été testés. Dans l'immense majorité des cas, aucun de ces gènes ne s'est révélé pertinent pour l'explication du diabète de type 2 chez l'homme.

La contribution de phénomènes génétiques multiples pour expliquer l'insulinorésistance et/ou le déficit insulinosécrétoire est actuellement bien démontrée : concordance beaucoup plus importante du diabète de type 2 chez les jumeaux homozygotes que chez les jumeaux dizygotes, prévalence majeure du diabète 
de type 2 dans certains groupes ethniques (Indiens Pimas, îles du Pacifique Sud), diminution de la prévalence du diabète de type 2 dans ces groupes ethniques quand ils sont "métissés". L'établissement, sur de grands groupes, de I'héritabilité du diabète de type 2 montre clairement que des gènes multiples sont impliqués. Leur nombre et leurs rôles respectifs sont en passe d'être décrits, néanmoins la complexité du problème est considérable : certains gènes peuvent interagir entre eux, certains gènes peuvent contribuer à une anomalie métabolique susceptible d'être diabétogénique dans un seul phénotype, certains gènes ne peuvent être impliqués qu'à l'état homozygote. Enfin, et surtout, la susceptibilité génétique et les facteurs environnementaux (entendu au sens large) peuvent interagir de façon très diverse. Par exemple, l'excès calorique pourrait réguler des gènes impliqués dans la sensibilité à l'insuline et le vieillissement des gènes impliqués dans la sécrétion d'insuline. Du fait du rôle majeur des facteurs environnementaux, des diabètes de type 2 pourraient être essentiellement non génétiques et des diabètes de type 2 pourraient ne pas apparaître chez des individus très "susceptibles " parce qu'ils n'ont pas été exposés à ces facteurs. Les développements très récents de l'épigénétique dans ces études seront peutêtre un élément déterminant de la compréhension des mécanismes liant gènes, environnement, hygiéno-diététique et diabète de type 2 .

\subsection{Insulinorésistance et diabète de type 2}

Nous avons abordé son importance dans le chapitre obésité. II nous faut souligner ici que, bien que l'insulinorésistance soit un phénomène très précoce dans le diabète de type 2 , celle-ci peut exister de façon très prononcée sans diabète de type 2 authentique. Les obèses morbides ont une insulinorésistance majeure du muscle et du tissu adipeux alors que le diabète de type 2 n'atteint pas plus de $10 \%$ de ces patients et celui-ci est exceptionnellement sévère. À I'inverse, des patients avec une obésité beaucoup moins sévère mais androïde et une insulinorésistance similaire ont très souvent un diabète de type 2 sévère.

Les modèles de souris transgéniques nous ont appris que les souris avec $\mathrm{KO}$ du récepteur à l'insuline dans le muscle présentent une insulinorésistance majeure et une discrète intolérance au glucose. En revanche, les souris KO pour le transporteur du glucose Glut IV, dont l'activité est strictement insulinodépendante, présentent une insulinorésistance et un diabète rapidement sévère.

Il a été récemment démontré qu'au niveau moléculaire, l'insulinorésistance du muscle du patient diabétique de type 2 était spécifique, c'est-à-dire différente de l'insulinorésistance de l'obèse non diabétique. Malheureusement, à ce jour, aucune méthode ne permet de caractériser in vivo cette spécificité. En clair, rien, au niveau clinique, ne permet de différencier l'insulinorésistance d'un patient obèse non diabétique de l'insulinorésistance d'un patient présentant une obésité 
similaire avec un diabète de type 2. Il faudra probablement encore de longues années pour que l'on puisse caractériser au niveau phénotypique ces différences décelées au niveau cellulaire.

\subsection{Insulinosécrétion et diabète de type 2}

De nombreux éléments plaident aujourd'hui en faveur d'un rôle précoce, voire initial, d'un déficit insulinosécrétoire dans la genèse du diabète de type 2 . La démonstration d'un rôle spécifique et précoce du déficit insulinosécrétoire chez le diabète de type 2 a été longue et difficile pour, au moins, trois raisons :

- le déficit insulinosécrétoire, même à la phase tardive du diabète de type 2, n'est jamais total, c'est-à-dire qu'il persiste toujours une insulinosécrétion basale ;

- le déficit ne porte que sur le couplage entre métabolisme du glucose dans la cellule $\beta$ et l'insulinosécrétion ;

- sécrétion d'insuline et sensibilité à l'insuline sont intimement couplées et évoluent de façon parallèle avec l'âge sans que le lien en cause dans ce couplage et ce parallélisme soient clairement établis.

Malgré ces difficultés, de nombreux arguments chez l'homme et l'animal permettent d'affirmer que le déficit insulinosécrétoire est probablement initiateur du diabète de type 2. Il est maintenant établi que la perte du pic précoce d'insulinosécrétion et de sa pulsatilité en réponse au glucose est caractéristique de la phase initiale du diabète de type 2. De plus, in vitro, l'exposition chronique des îlots de Langerhans à un excès de glucose et/ou d'acides gras entraîne une diminution de l'insulinosécrétion et une augmentation de l'apoptose. Ces observations ont été à l'origine du concept de "glucolipotoxicité ». Elles expliquent pourquoi les îlots de Langerhans prélevés sur des pancréas de sujets diabétiques de type 2 sécrètent, après deux semaines de culture, moins $d^{\prime}$ insuline que les îlots de pancréas d'un sujet normal. De nombreux autres arguments, étayant ou contredisant le rôle du déficit insulinosécrétoire, ont permis de mettre en évidence l'importance de la plasticité de la fonction bêtacellulaire. Cette plasticité est non seulement fonctionnelle, mais aussi histologique, chiffrée par le nombre $\mathrm{d}^{\prime}$ îlots et le nombre de cellules $\beta$. Cet élément, très clair chez l'animal, pourrait être identifié chez l'homme dans un proche avenir par les développements en cours de l'imagerie fonctionnelle et quantitative des îlots de Langerhans et/ou de la cellule $\beta$. 


\subsection{Complications micro- et macro-angiopathiques du diabète de type 2}

La description de l'ensemble de ces complications a été clairement établie par les cliniciens dans les années 1950. Néanmoins, seules les données épidémiologiques des vingt dernières années ont permis de déterminer le retentissement majeur du diabète de type 2 sur les évènements cardiovasculaires souvent mortels. Les mécanismes de la glucotoxicité (reins, rétine, nerfs) ont été décryptés avec la mise en évidence d'un rôle important d'un dysfonctionnement mitochondrial et d'une activation de la protéine kinase $C$.

La morbidité induite par ces complications va probablement beaucoup évoluer dans les années à venir. En effet, durant les vingt dernières années, on a vu, suite aux efforts des sociétés savantes, des pouvoirs publics et de l'industrie pharmaceutique, se développer de nouveaux outils thérapeutiques et une " prise en charge agressive » des diabètes de type 2. Aujourd'hui, on peut affirmer que :

- la glycémie peut et doit être contrôlée par les antidiabétiques oraux et/ou I'insulinothérapie ;

- I'hypertension artérielle peut et doit être contrôlée par les nombreuses familles de médicaments antihypertenseurs;

- la dyslipidémie et l'athérome peuvent et doivent être contrôlés par les efforts hygiénodiététiques et/ou les « hypocholestérolémiants » largement disponibles et totalement pris en charge;

- la néphropathie diabétique peut et doit être arrêtée par la mise en route précoce des inhibiteurs du système rénine angiotensine.

- les phénomènes de thrombose à l'origine des accidents cardiaques et cérébraux peuvent et doivent être prévenus par l'utilisation de l'aspirine ;

- la rétinopathie proliférante peut et doit être traitée par photocoagulation dès les prémices de son apparition.

Cette déclinaison, peut-être fastidieuse quoique incomplète, devrait permettre de penser que le traitement du diabète de type 2 est, aujourd'hui, maîtrisé. II $\mathrm{n}^{\prime}$ en est rien et une coordination médicale d'envergure sera nécessaire pendant de longues années pour que les réticences et/ou échappements thérapeutiques soient vaincus. 
Parmi les nombreux facteurs d'échec, on peut noter :

- l'extrême difficulté de prendre de façon "observante » 15 à 25 comprimés par jour. Le développement d'association thérapeutique est un impératif;

- le diabète de type 2 touche de plus en plus les populations les plus défavorisées qui n'ont pas, bien souvent pour des raisons culturelles, accès à une médecine raisonnée, scientifique, souvent éloignée de la préoccupation de son bien-être par le patient ;

- la mise en œuvre de ces traitements nécessite les efforts coordonnés des médecins référents, des spécialistes (diabétologues, néphrologues, cardiologues, ophtalmologues) dont le nombre est, malheureusement, encore restreint pour de longues années;

- le coût et l'importance "psychique » de la prise en charge obligera à la définition d'objectifs thérapeutiques successifs négociés pas à pas au fil des ans, entre le patient et son médecin.

Ces perspectives " attractives 》 devraient contribuer à de nouvelles décisions politiques concernant une thérapie préventive du diabète de type 2 et de l'obésité. Les problématiques des organismes d'assurance maladie, publics ou privés de " prédiction génétique » du diabète et de l'obésité renvoient aux mythes inquiétants de la toute puissance médicale et de la «normalisation » d'un nombre considérable de sujets. Les stratégies d'intervention médicale et raisonnée sur le comportement alimentaire et l'activité physique, bien souvent tenues en échec, doivent être maintenant envisagées sous un angle économique et sociétal plutôt que médical.

\section{7 Évaluation des politiques scientifiques, de santé publique et des industries pharmaceutiques}

La politique scientifique, dans le domaine de l'obésité et du diabète de type 2, a clairement contribué à l'apparition d'un niveau d'excellence de la recherche française. Tant en termes de quantité que de qualité, la France est dans le groupe des cinq premières nations dans le monde. Les financements additionnels conséquents obtenus grâce aux contrats avec la nouvelle Agence nationale de la recherche contribuent à la création en cours de centres français de plus en plus cohérents. On peut citer, parmi beaucoup d'autres :

- la confirmation de l'envergure des équipes parisiennes dans le domaine de l'obésité morbide, de la génétique et de l'épigénétique de l'obésité, du mécanisme d'action de l'insuline sur le foie et le tissu adipeux, des relations entre poids corporel, consommation alimentaire des Français et 
industries agroalimentaires, de l'épidémiologie des maladies cardiovasculaires associées au diabète ;

- le développement des compétences en génétique du diabète de type 2 et de l'obésité à Paris et à Lille et Toulouse;

- les performances dans le domaine des relations entre dyslipoprotéinémie et athérome à Paris, Dijon, Lille, Lyon, Marseille ;

- les travaux des équipes autours du CRNH de Lyon sur les mécanismes géniques de l'insulinorésistance chez l'homme, sur la signalisation cérébrale des signaux post-prandiaux dans la veine porte, les relations obésitécancer ;

- les travaux des équipes parisiennes et niçoises sur la signalisation insulinique et le transport du glucose ;

- les travaux sur la prévention de l'obésité par l'activité physique chez les adolescents de la région strasbourgeoise.

Malgré cette qualité et ce développement significatif de l'activité de recherche, des domaines très importants de la recherche sur le diabète et l'obésité sont absents ou seulement en émergence. On peut citer :

- la neurophysiologie du comportement alimentaire étudié dans bien peu d'équipes (Paris et Dijon) ;

- I'insulinosécrétion tant au niveau physiologique que biochimique, effectivement maîtrisée par seulement une ou deux équipes à Paris ;

- seulement trois équipes (Paris, Lyon et Strasbourg) qui ont eu la capacité et la compétence suffisantes pour être leader dans trois essais thérapeutiques en relation avec l'obésité, la nutrition et les pathologies cardiovasculaires. Aucune d'entre elles n'est leader dans des essais thérapeutiques de taille européenne ou mondiale.

Au niveau de la santé publique, les actions conjointes de l'Inserm, de l'Inra, des grandes mutuelles, de la Direction générale de la santé, ont contribué, de façon majeure, à la diffusion de messages clairs sur le diabète et l'obésité auprès du grand public et des acteurs de la santé. Ces actions, très conséquentes au niveau financier, doivent être évaluées avec l'ensemble des acteurs par des experts extérieurs afin de mieux identifier les cibles de la prévention. II est possible, voire probable, que le discours raisonnable et raisonné sur la prévention de l'obésité par l'activité physique et une alimentation saine et équilibrée, devra trouver d'autres modes d'expression mettant en jeu, notamment, les mythes et 
les symboles inscrits dans la notion de faim, d'appétit, de plaisir alimentaire. II devra aussi s'inscrire dans les programmes éducatifs dès le plus jeune âge. La nouvelle loi sur la santé publique mettra, peut-être, en place les éléments d'une telle réflexion qui devra impérativement sortir des sentiers battus.

L'industrie pharmaceutique joue et jouera un rôle majeur dans ces évolutions. Elle finance, actuellement, très largement des recherches très ciblées au niveau moléculaire pour trouver le ou les quelques protéines qui pourraient jouer le rôle de chef $d^{\prime}$ orchestre de la genèse du diabète de type 2 . Ce rêve, en relation avec la mondialisation de ces groupes industriels, est peut-être fantasmatique. II est possible, voire probable, que les thérapeutiques du diabète de type 2 auront à s'adapter aux caractéristiques socioculturelles des patients. Les interactions gènes-nutriments en cours de décryptage et le fond génétique des populations, lui aussi à l'étude, pourraient modifier profondément certaines attitudes thérapeutiques.

\section{Recommandations}

- Poursuivre les actions de regroupement des divers EPST sur la problématique obésité et diabète de type 2 avec, pour objectif, de soutenir, d'une part, des actions de recherche d'envergure européenne et, d'autre part, de faire émerger de petites équipes de pointe sur des stratégies et des hypothèses originales. II faut noter que la créativité des équipes est souvent modulée, voire bridée, par les choix financiers des industries pharmaceutiques dont les cibles de recherche visent plus souvent une valorisation à court terme qu'un développement original et risqué.

- Définir quelques axes radicalement nouveaux permettant des regroupements thématiques originaux. Parmi ceux-ci, on pourrait insister sur :

- nutrition et cerveau en tirant partie des compétences nationales en neurosciences et en nutrition;

- imagerie métabolique pour quantifier les cellules bêta, les différents types de dépôts lipidiques, la stéatose hépatique, en combinant les acquis de la biologie cellulaire et les nouveaux outils de l'imagerie par fluorescence in vivo chez l'animal et l'homme.

- Proposer des moyens à deux ou trois centres français lavec une aide conséquente des centres hospitaliers, des mutuelles et des organismes de Sécurité sociale), d'organiser et de réaliser des projets de prévention primaire de l'obésité andrö̈de dans les populations défavorisées et/ou les apparentés de patients diabétiques de type 2 .

- Soutenir la création d'unités mixtes de recherche entre CHU et EPST en gardant le même niveau de recherche de l'originalité et de l'excellence. 
- De telles évolutions ne seront pas possibles sans la mise en place de structures administratives communes et simplifiées. La multiplicité incontournable des partenaires dans ces constructions sera certainement source d'une complexité de gestion incompatible avec le bon fonctionnement de groupes de recherche. Là aussi il faut donc inventer.

\section{Références bibliographiques}

Basdevant A., Laville M. et Lerebours E. (2001). Traité de nutrition clinique de l'adulte. Médecine Sciences Flammarion, Paris.

Grimaldi A. (2005). Traité de diabétologie. Médecine-Sciences Flammarion, Paris.

Kahn CR., Weir GC., King GI., Jacobson AM., Moses AC. et Smith R.J. (2005). Joslin Diabetes Wellitas. $14^{\text {th }}$ ed. Lippincott Williams and Wilkins.

Haeffmer N. (2005). Recherches sur le diabète en France. Données bases web of Sciences Inserm Paris.

Kahn R., Buse J., Ferrannini E. et Stern M. (2005). The Metabolic Syndrome: time-for a critical appraisal. Diabetes Care, 28 : 2289-2304.

\section{Remerciements}

Jean-Paul Riou remercie Martine Laville, Chantal Simon et Hubert Vidal pour la relecture critique de ce rapport.

\section{Abréviations}

Afssaps : Agence française de sécurité sanitaire des produits de santé AgRP : agouti-related protein

CART : cocain and amphetamine related transcript

CRF : corticotrophin-releasing factor

HTA : hypertension artérielle

IL-6 : interleukine 6

LADA : latent autoimmune diabetes in adult

$M C 4 R$ : récepteur des mélanocortines de type 4

MODY : matury onset diabetes of the youth

MSH : melanocyte stimuling hormone

PPAR : peroxisome proliferator-actived receptor

PYY : peptide YY

TA : tissu adipeux

TNF : tumor necrosis factor 
